\section{Two cases of placenta accreta identified during pregnancy after laparoscopic myomectomy and resection of adenomyosis}

\author{
Shigetaka Matsunaga, Takahiro Uotani, Ken Ohara, Yasushi Takai, \\ Kazunori Baba, Hiroyuki Seki
}

Center for Maternal, Fetal and Neonatal Medicine, Saitama Medical Center/Saitama Medical University, Saitama, Japan
Reprint request to:

Shigetaka Matsunaga, M.D., Ph.D., Center for Maternal, Fetal and Neonatal Medicine, Saitama Medical Center/Saitama Medical University, 1981 Kamoda, Kawagoe, Saitama, 350-8550, Japan.

E-mail: shige_m@saitama-med. ac.jp

\section{Key words:}

adenomyomectomy, laparoscopic myomectomy, placenta accreta, prior myomectomy, ultrasonography

Received: December 26, 2014

Revised: February 23, 2015

Accepted: March 11, 2015

DOI:10.14390/jsshp.3.38

We report two cases of placenta accreta identified during pregnancy after laparoscopic surgery. The first case involved a 39-year-old woman with a history of miscarriage. Ultrasonography and MRI revealed multiple uterine myomas and adenomyosis. One year after laparoscopic myomectomy and adenomyosis resection, ultrasonography at 33 weeks of gestation revealed a thin uterine posterior wall and placental invagination, indicating placenta accreta. During cesarean section, the muscular layer required repair after removal of the placenta. The second case involved a 37-year-old woman with excessive menstruation. Ultrasonography revealed a large uterine myoma adjacent to the endometrial cavity. Four years after laparoscopic myomectomy, ultrasonography revealed placenta accreta at 35 weeks of gestation. During cesarean section, the muscular layer needed to be partially resected together with the placenta. A thorough placental evaluation with ultrasonography is recommended in pregnancies achieved after myomectomy and adenomyosis resection.

\section{Introduction}

The uterine endometrium can be damaged during laparoscopic myomectomy ${ }^{1)}$ and adenomyosis resection (adenomyomectomy). Postoperatively, the placenta can invade the myometrium at the resection site. If the intrauterine cavity is not normalized despite a sufficient contraceptive period, placenta accreta may occur in the subsequent pregnancy. Placenta accreta can result in severe maternal hemorrhage and, occasionally, maternal morbidity and death. ${ }^{2)}$ Here we report our experiences with two pregnant women with placenta accreta occurring after laparoscopic myomectomy and adenomyomectomy.

\section{Case report}

A 39-year-old woman (case 1: gravida 1, para 0) with a history of miscarriage was admitted to our hospital. Ultrasonography (USG) examination and MRI revealed multiple uterine myomas (maximum diameter, $7 \mathrm{~cm}$ ) and adenomyosis. After gonadotrophic-releasing hormone agonist (GnRHa) was administered, laparoscopic myomectomy and adenomyomectomy were performed. To reduce intraoperative hemorrhage, a $200 \times$ dilution of vasopressin in physiological saline was locally injected using an $18 \mathrm{G}$ needle. The posterior wall of the uterus was vertically incised, and the adenomyosis was removed using the convex-lens method. ${ }^{3)}$ Damage to the uterine endometrium (the uterine cavity was open approximately $1 \mathrm{~cm}$ ) was found intraoperatively, but sufficient closure was achieved. The myometrium was closed in three layers using a 1-0 Monocryl (Ethicon Inc., Somerville, U.S.A.), with a purse-string suture carried out to avoid creating dead space in the deepest part of the myometrial layer.

Since USG performed four months postoperatively revealed a normalized myometrium and endometrium, the patient was informed that she can proceed to become pregnant. Spontaneous pregnancy occurred six months postoperatively and cesarean section was scheduled 


\section{A}

B

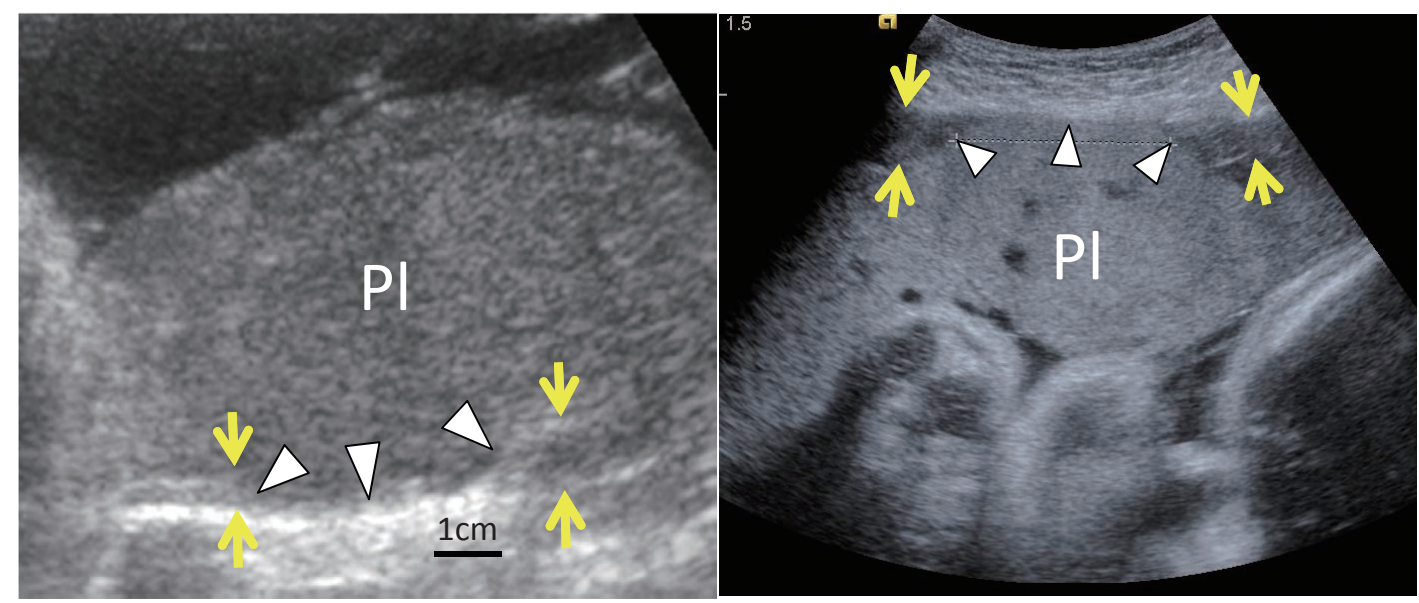

Figure 1. High resolutional ultrasonographies of placenta accreta in the third trimester.

(A) Ultrasonography (USG) of case 1 at 33 weeks of gestation. The placenta (PI) was located in the posterior wall of the uterus. Myometrial thinning (up to $4 \mathrm{~cm}$; arrows) and placental invagination were visible (arrowheads).

(B) USG of case 2 at 35 weeks of gestation. The placenta (PI) is located in the anterior wall of the uterus. Placental invagination (arrowheads) was observed at the site of myometrial thinning (arrows).

A

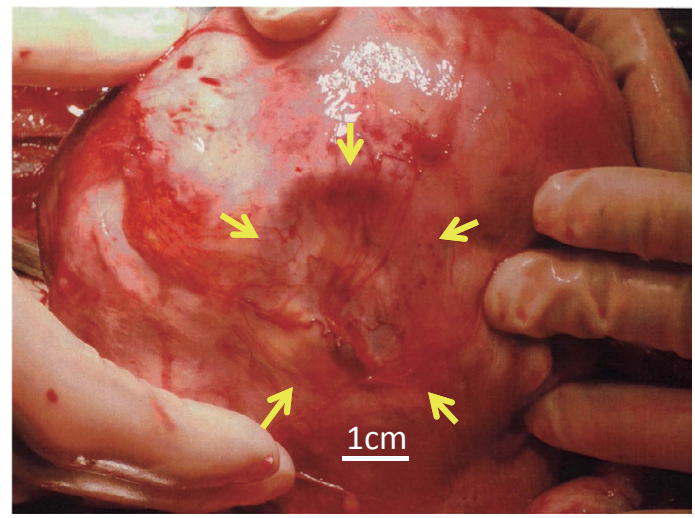

C

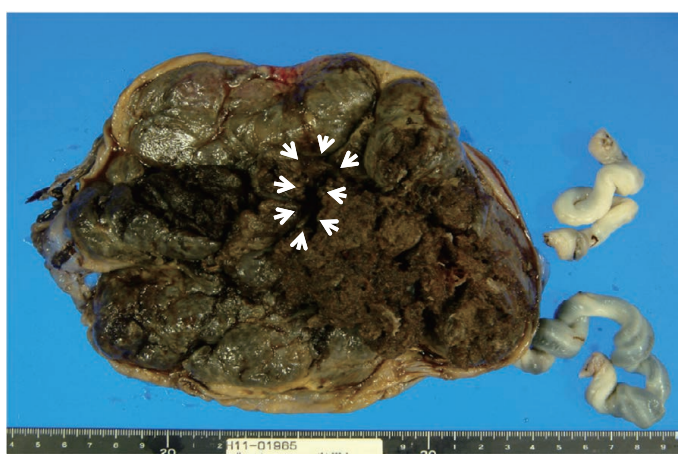

B

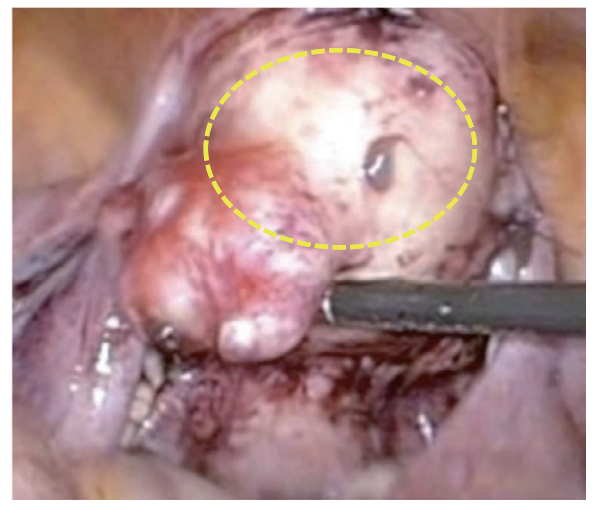

D

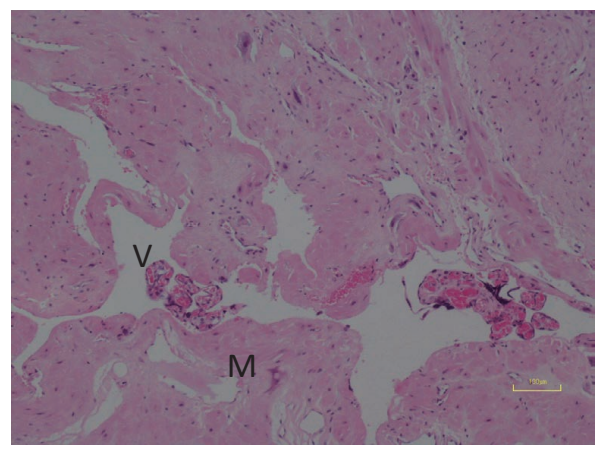

Figure 2. The macroscopic and microscopic findings for diagnosis of placenta accreta.

(A) A recessed area (arrows) in the posterior uterine wall was observed upon cesarean section in case 1. (B) Adenomyosis was observed (circle) at the same location in laparoscopic surgery for case 1. (C) Macroscopic image of the placenta in case 1. A defect (arrowheads) in the placenta was caused by its removal from the attached uterine wall. (D) Microscopic images of the placenta in case 2. HE staining. The myometrium (M) was directly adjacent to the placental villi (V) without the decidua. 
due to previous myomectomy and adenomyomectomy. USG performed at 33 weeks of gestation demonstrated myometrial thinning (up to $4-\mathrm{cm}$ ) and invagination of the placenta in the posterior wall (Figure 1A), indicative of placenta accreta. Cesarean section performed at 37 weeks and 5 days of gestation revealed myometrial thinning and placenta accreta at the site of the previous adenomyomectomy (Figures 2A, 2B). After the placenta was removed to the extent possible, the myometrium was repaired. Intraoperative blood loss was $1,840 \mathrm{ml}$; 10 units of packed red blood cells and 10 units of fresh frozen plasma were transfused. Macroscopic examination of the placenta revealed a defect due to its adhesion to the myometrium (Figure 2C). Given the lack of decisive microscopic findings, the postoperative diagnosis was assumed to be clinical placenta accreta.

A 37-year-old woman (case 2: gravida 1, para 1) had undergone cesarean section at 37 weeks of gestation owing to a myoma-complicated pregnancy, and was admitted due to excessive menstruation. On USG and MRI examination, an intramural myoma (approximately $8 \mathrm{~cm}$ in diameter) was identified in the anterior uterine wall adjacent to the endometrial cavity. After GnRHa was administered, laparoscopic myomectomy was performed. No obvious endometrial damage was found intraoperatively, and the myometrium was closed in three layers using a 1-0 Monocryl. Spontaneous pregnancy was achieved four years postoperatively. USG performed at 35 weeks of gestation revealed that the attachment site of the placenta was remote from the cesarean section scar in the uterine wall. However, placenta accreta was highly suspected because a placental invagination was observed at the location of the previous myomectomy (Figure 1B).

Cesarean section performed at 37 weeks and 6 days of gestation revealed a deformed uterine wall and placenta accreta. The placenta was removed together with the myometrium, which required repair. Intraoperative blood loss was $1,500 \mathrm{ml}$, and $600 \mathrm{ml}$ autologous transfusion was performed. Postoperative pathological examination revealed placental villi directly adjacent to the myometrium (Figure 2D), leading to the postoperative diagnosis of pathological placenta accreta.

\section{Discussion}

Placenta accreta occurs at an incidence of $1 / 540$ to $1 / 93,000,{ }^{4,5}$ and can result from placenta previa, previous cesarean section, endometrial curettage, hysteroscopy, transarterial embolization, and myomectomy. ${ }^{6}$ At our institution, 1,796 patients underwent cesarean sections from January 1, 2008 to March 31, 2011, and 31 (1.7\%) of these patients were diagnosed with placenta accreta intraoperatively (Table 1). Placenta accreta was observed primarily at the scar of previous cesarean sections
Table 1. Potential risk factors of placenta accreta

\begin{tabular}{lc}
\hline Potential risk factors of placenta accreta & $n$ \\
\hline Previous cesarean section & 20 \\
After laparoscopic myomectomy, & 2 \\
adenomyomectomy & 2 \\
After abdominal myomectomy & 2 \\
After TAE & 1 \\
After resection of the uterine septum & 1 \\
After D\&C & 1 \\
ICSI-ET & 2 \\
Others & 31 \\
Total
\end{tabular}

Analysis of 31 cases of placenta accreta diagnosed during cesarean section in our center. "Others" in the list involved resection of an endocervical polyp. TAE, transcatheter arterial embolization; D\&C, dilatation and curettage; ICSI-ET, intracytoplasmic sperm injection and embryo transfer.

(20 cases). ${ }^{7)}$ However, placenta accreta following the resection of myomas and adenomyosis occurred in 4 of the 31 cases $(12.9 \%)$, including the present two laparoscopic cases. In these two cases, laparoscopic myomectomy and adenomyomectomy may have caused placenta accreta, since it was found in the exact location of the surgery (Figures 1A, 1B, 2A, 2B). Moreover, because the adenomyosis and myomas were found adjacent to the endometrial cavity in both cases, placenta accreta was possibly due to a failure of endometrial regeneration following scar formation and circulatory failure caused by the excision and suturing of the myometrium. ${ }^{8)}$ Additionally, since the decidua was thinner than the normal decidua in case 2 , abnormal invasion of the villi may have resulted from the implantation of the embryo. ${ }^{9)}$

Intrusion of the placenta into a thinning or defective myometrium is considered an important finding of an invasive placenta; however, this evaluation is likely better performed with high resolution USG rather than MRI. ${ }^{10,11)}$ In the present two cases, MRI examination did not reveal either finding, but placenta accreta was strongly suspected because USG clearly revealed these findings (Figure 1A, 1B). With sufficient preoperative planning, including autologous transfusion, cesarean section can be successfully carried out. In these two cases, hysterectomy would have reduced the amount of hemorrhage, but since evaluating the extent of placenta accreta was possible using USG, more flexibility was provided to the operator prior to cesarean section.

To our knowledge, the present paper reports the first two cases of placenta accreta resulting from laparoscopic myomectomy or adenomyomectomy. Indeed, Gyamfi- 
Bannerman et al. reported that no placenta accreta occurred in their 176 myomectomy cases and concluded that prior myomectomy is not associated with placenta accreta. ${ }^{7}$ Prior to surgery, patients may need to be informed about the postoperative risk of placenta accreta in subsequent pregnancies, and sufficient information should be provided to obstetricians in charge of pregnancy and postpartum management. Finally, in cases of prior myomectomy or adenomyomectomy, the placenta should be evaluated using high resolution USG prior to delivery. MRI may be useful for diagnosing placenta accreta at the posterior uterine wall in selected cases.

\section{Acknowledgements}

We thank all individuals who contributed to this study.

\section{Conflict of interest}

None.

\section{References}

1. Casini ML, Rossi F, Aqostini R, Unfer V. Effect of the position of fibroids on fertility. Gynecol Endocrinol. 2006; 22: 106-109.

2. Kayem G, Deneux-Tharaux C, Sentilhes L; PACCRETA group.
PACCRETA: clinical situations at high risk of Placenta ACCRETA/ Percreta: impact of diagnostic methods and management on maternal morbidity. Acta Obstet Gynecol Scand. 2013; 92: 476482.

3. Ota H, Hada T, Matsuura T, et al. Laparoscopic resection of adenomyosis in this hospital: convex lens resection. Journal of Japan Society of Endometriosis. 2008; 29: 85-90. (In Japanese.)

4. Fox H, Elston CW. Pathology of the placenta. Major Probl Pathol. 1978; 7: 1-491.

5. Bauer ST, Bonanno C. Abnormal placentation. Semin Perinatol. 2009; 33: 88-96.

6. Miller DA, Chollet JA, Goodwin TM. Clinical risk factors for placenta previa placenta accreta. Am J Obstet Gynecol. 1997; 177: 210-214.

7. Gyamfi-Bannerman C, Gilbert S, Landon MB, et al. Risk of uterine rupture and placenta accreta with prior uterine surgery outside of the lower segment. Obstet Gynecol. 2012; 120: 1332-1337.

8. Garmi G, Goldman S, Shalev E, Salim R. The effects of decidual injury on the invasion potential of trophoblastic cells. Obstet Gynecol. 2011; 117: 55-59.

9. Khong TY. The pathology of placenta accreta, a worldwide epidemic. J Clin Pathol. 2008; 61: 1243-1246.

10. Murayama Y, Baba K, Seki H. Abnormality and imaging diagnosis during second trimester of pregnancy. Obstetrical and Gynecological Practice. 2008; 57: 421-427. (In Japanese.)

11. Jauniaux E, Toplis PJ, Nicolaides KH. Sonographic diagnosis of a non-previa placenta accreta. Ultrasound Obstet Gynecol. 1996; 7: $58-60$. 